EPJ Web of Conferences 114,02138 (2016)

DOI: 10.1051/epjconf/201611402138

(C) Owned by the authors, published by EDP Sciences, 2016

\title{
Interaction of two circular cylinders in the flow field at low Reynolds numbers
}

\author{
Rut Vitkovičová ${ }^{1 a}$, Vladislav Skála ${ }^{1}$ \\ ${ }^{1}$ CTU in Prague, FME, Department of Fluid Dynamics and Thermodynamics, Technická 4, 16607 Prague, Czech Republic
}

\begin{abstract}
A set of points of a velocity fluctuation was measured in the wake behind the two circular cylinders by CTA method. Measurements were carried out for several positions of the secondary cylinder in the wake of primary cylinder and for a set of modes of low Reynolds number (Re max 2600). The goal was to obtain a set of data and information about the influence of the secondary cylinder on the primary cylinder.
\end{abstract}

\section{Introduction}

Investigating the mutual influence of two or more cylinders in the flow field is the theme of many works in the field of fluid mechanics and related fields, but nevertheless it is still an open problem which is not fully resolved. Understanding the mutual influence of cylinders in the flow field allows us among other things, to understand the phenomena taking place in geometrically similar applications in technology, e.g. construction (e.g. the chimney towers), engineering etc., and also allows to find areas affecting the wake of the dominant cylinder.

M. M. Zdravkovich in his review [1] deals with an observation of an influent of two cylinders in a configuration tandem and a staggered arrangement. Hwang and Choi introduce in [2] with works that deal with a control of wake on the one hand passively and other actively. They comparing the area of influence of the secondary cylinder on primary cylinder in research Strykowski and Sreenivasan in [3] with the results of Hwang and Choi [4]. D. Sumner in his article [5] clearly summarizes several studies by authors engaged in research of wake behind a cylinder in a number of settings and gives an overview of the performed measurements, measuring apparatuses and measuring stands. Overview of the measured dependence Strouhal number ratio gap between the cylinders in a parallel arranged and comparing them with the results of other authors is given in a paper from Yokoi [6].

This article describes an experimental measurement of the influence of cylinders in the flow field in staggered configuration using the CTA method and presents the results of these measurements. This paper follows the measurements published in [7].

\section{Measurements}

\subsection{Experimental tunnel and measurement techniques}

The measurement by method CTA carried out in an Eiffeel type wind tunnel which has a speed range of approximately $0-20 \mathrm{~m} / \mathrm{s}$. The intensity of turbulence in the tunnel is around $1.1 \%$. A test section of the tunnel is closed. The length of the test section is $750 \mathrm{~mm}$ and its cross section is $300 \mathrm{~mm} \times 200 \mathrm{~mm}$. The test section is equipped with a movable system which enables automatic 3D positioning of the probe. Tunnel control, monitoring and data logging and control of movable system are performed by software written in LabView programming environment. The tunnel was fitted with a measuring system for the hot wire anemometers MiniCTA 54T30 (Dantec Dynamics) with 55P16 HW-probes for the measurements. The hot wire and cylinders are shown in Figure 1.

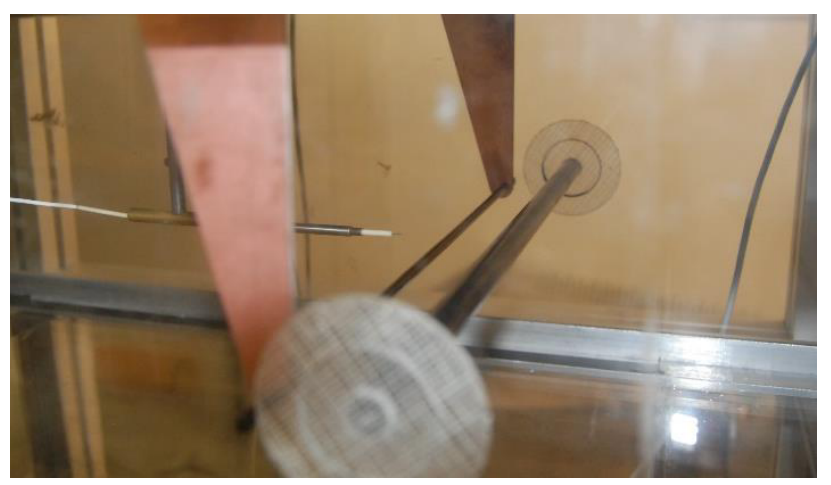

Figure 1. The hot wire probe and cylinders

\footnotetext{
${ }^{\mathrm{a}}$ Corresponding author: rut.vitkovicova@fs.cvut.cz
} 


\subsection{Experimental set-up}

Measuring method CTA was carried out for two pairs of cylinders, $\mathrm{D} / \mathrm{d}=1$ and $\mathrm{D} / \mathrm{d}=2.5$. The interaction of these cylinders was investigated for 7 (resp. 6) relative positions in the flow field, which were selected according to [2]. In Table 1 are given their coordinates and pitch ratios (R). The secondary cylinder was always located behind the primary cylinder. For each pair of cylinders were measured 16 modes of $\mathrm{Re}$ in the range of approximately from 400 to 2600 . For each mode Re was measured from 120 to 149 points placed in the wake of cylinders. Presented frequencies of vortex shedding was measured at a distance of $55-85 \mathrm{~mm}$ behind the primary cylinder. Sampling frequency of hot-wire probe was set to $30 \mathrm{kHz}$. In a Figure 2 is shown the set-up of the cylinders and coordinate system.

Table 1. Measured positions of secondary cylinder

\begin{tabular}{|c|c|c|c|}
\hline $\begin{array}{c}\text { Position } \\
\text { No. }\end{array}$ & $\mathbf{x} / \mathbf{D}$ & $\mathbf{y} / \mathbf{D}$ & $\mathbf{R}(\mathbf{m m})$ \\
\hline 1 & 4.3 & -1.25 & 44.8 \\
\hline 2 & 3.2 & -0.8 & 33.0 \\
\hline 3 & 2.4 & -0.8 & 25.3 \\
\hline 4 & 1.95 & -0.8 & 21.1 \\
\hline 5 & 2 & -0.6 & 20.9 \\
\hline 6 & 1.5 & -0.35 & 15.4 \\
\hline 7 & 1.5 & 0 & 15.0 \\
\hline
\end{tabular}

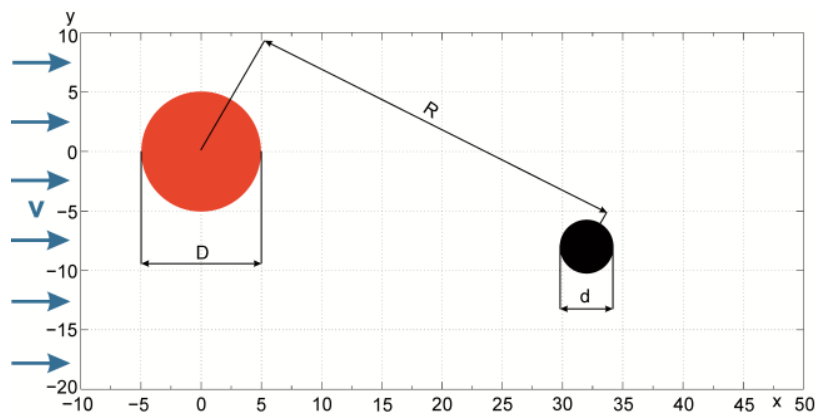

Figure 2. The set-up of the cylinders and coordinate system

\section{Results}

In the first step was obtained dominant vortex shedding frequencies from the measurement data for each mode of Re and the mutual position of the cylinders. In Figures 3 10 are compared a dependence of the frequency of vortex shedding on the R/D for the cylinders ratios 1 and 2.5. From these graphs it is clear that the vortex shedding frequency for ratio $\mathrm{D} / \mathrm{d}=1$ and thus the corresponding Strouhal number are lower than for the ratio of 2.5, except for positions 1, 2 and 7, which are in most cases approximately the same frequency. Positions 1 and 6 located outside of the areas of attenuating instabilities in [3] and [4], the positions 2, 3, 4 and 7 is located within these areas, and position 5 is located in the area defined in [4].

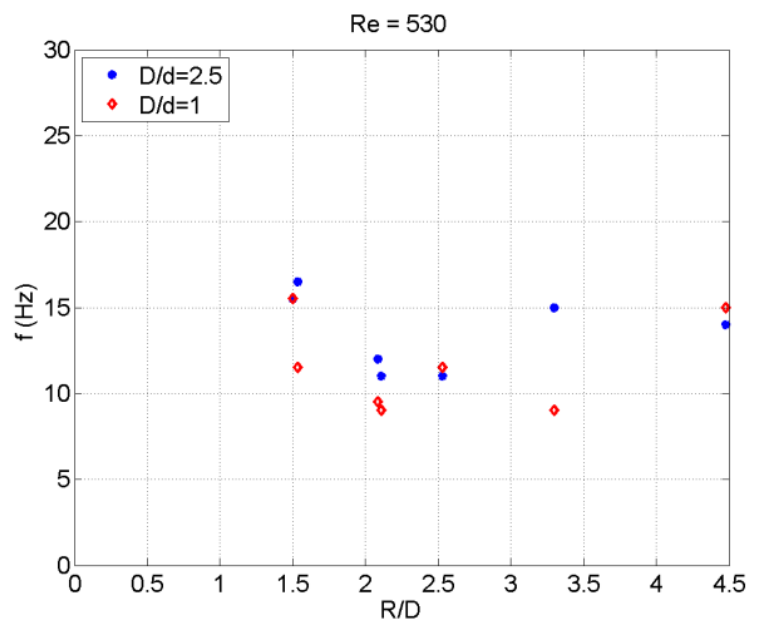

Figure 3. Relationship between the frequency and the pitch distance for $\operatorname{Re} 530$

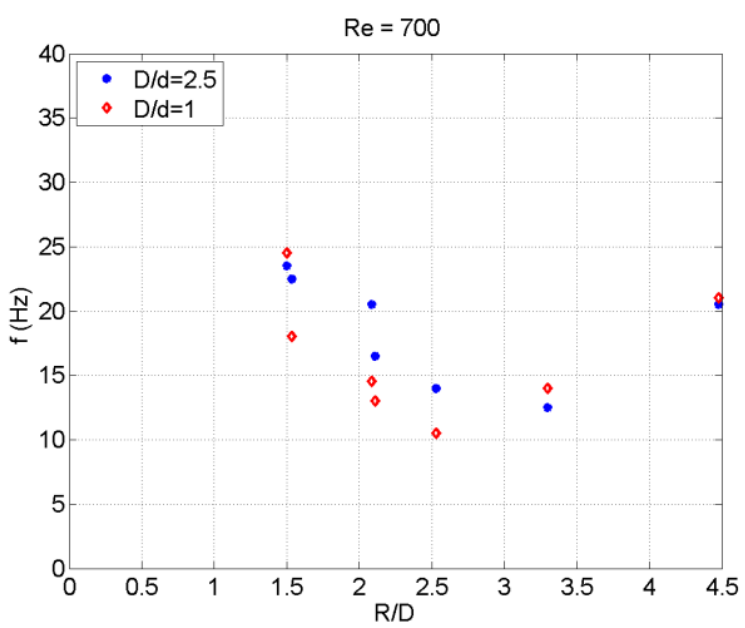

Figure 4. Relationship between the frequency and the pitch distance for Re 700

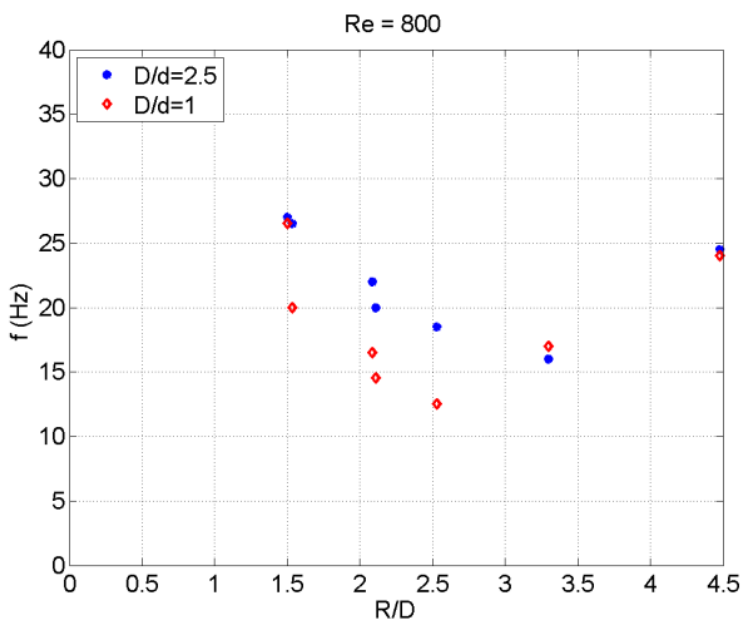

Figure 5. Relationship between the frequency and the pitch distance for Re 800 


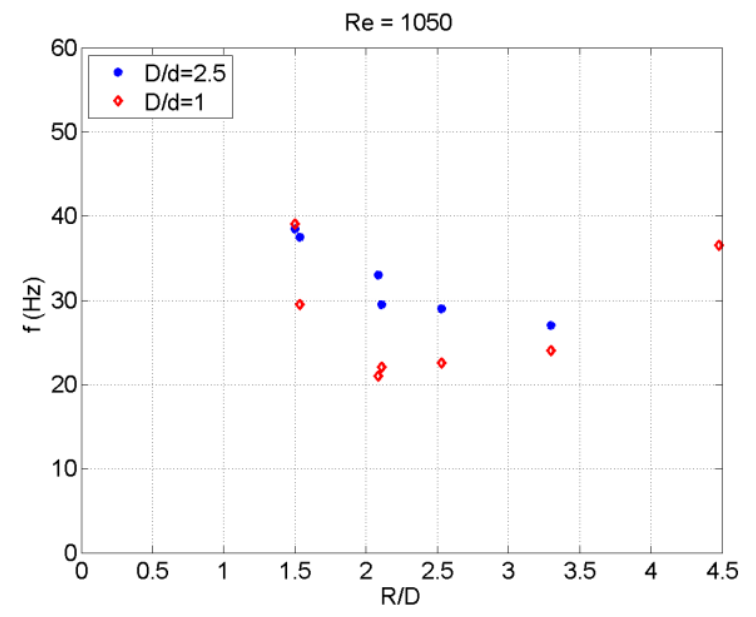

Figure 6. Relationship between the frequency and the pitch distance for Re 1050

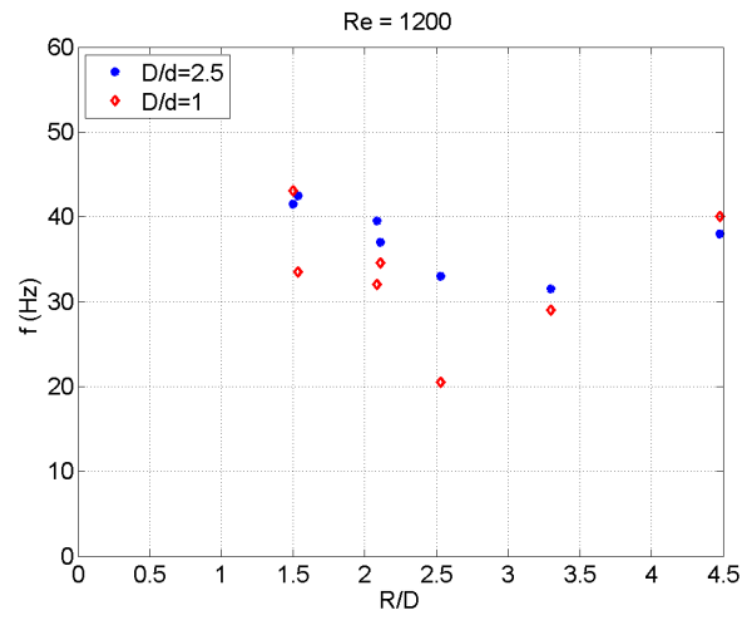

Figure 7. Relationship between the frequency and the pitch distance for Re 1200

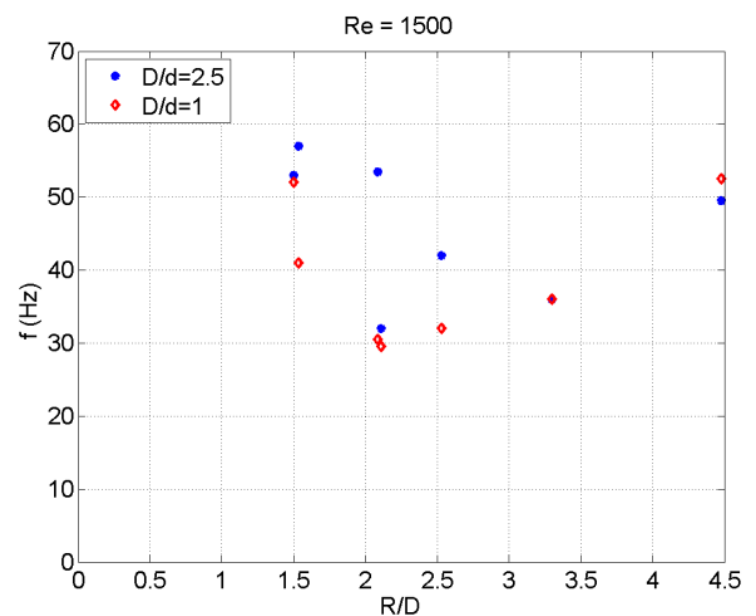

Figure 8. Relationship between the frequency and the pitch distance for Re 1500

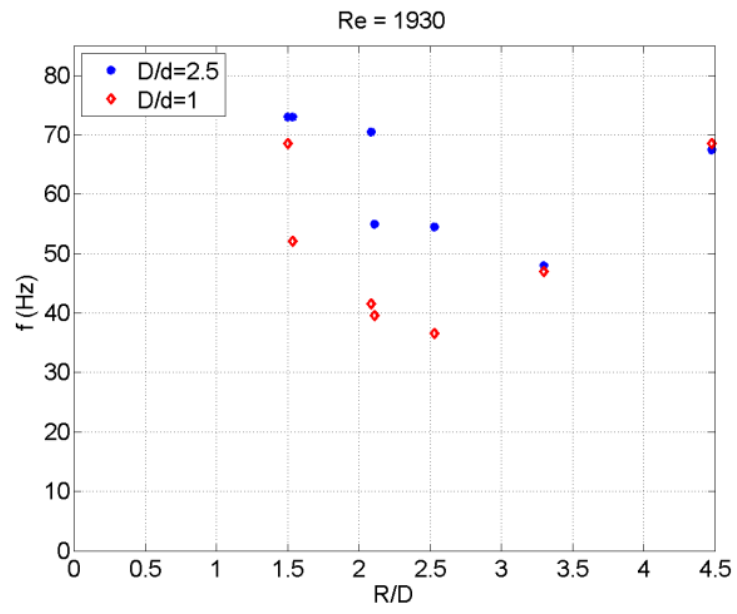

Figure 9. Relationship between the frequency and the pitch distance for Re 1930

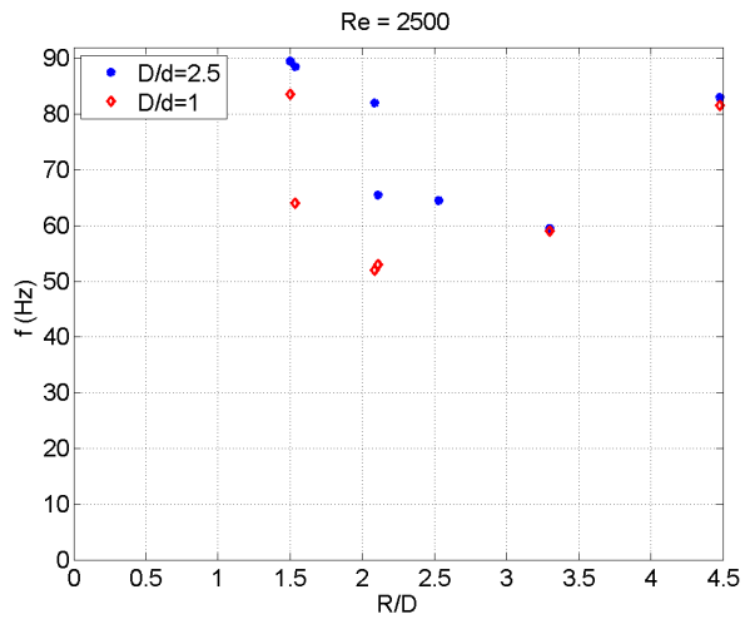

Figure 10. Relationship between the frequency and the pitch distance for Re 2500

Figures 11 and 12 compares the position of the secondary cylinder for a D / d ratio for all measurement modes Re. The obtained data show that for the positions 3 and 4 the smallest instabilities occur in the wake for both cylinders ratios. The next position with a low shedding frequency is position 2 for a ratio $\mathrm{D} / \mathrm{d}=2.5$, and for $\mathrm{D} / \mathrm{d}=1$ it is the position 5 . 


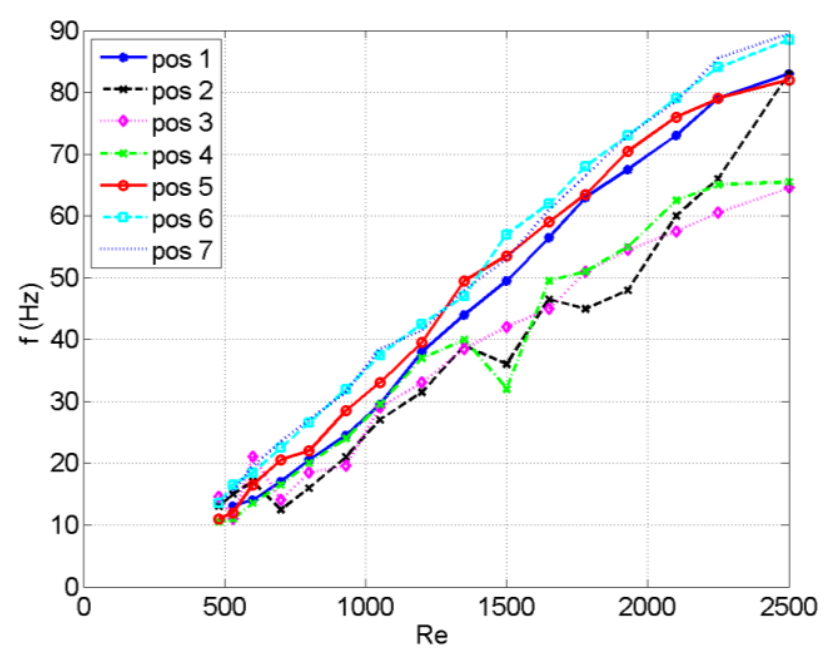

Figure 11. Relationship between the frequency and Reynolds numbers for $\mathrm{D} / \mathrm{d}=2.5$

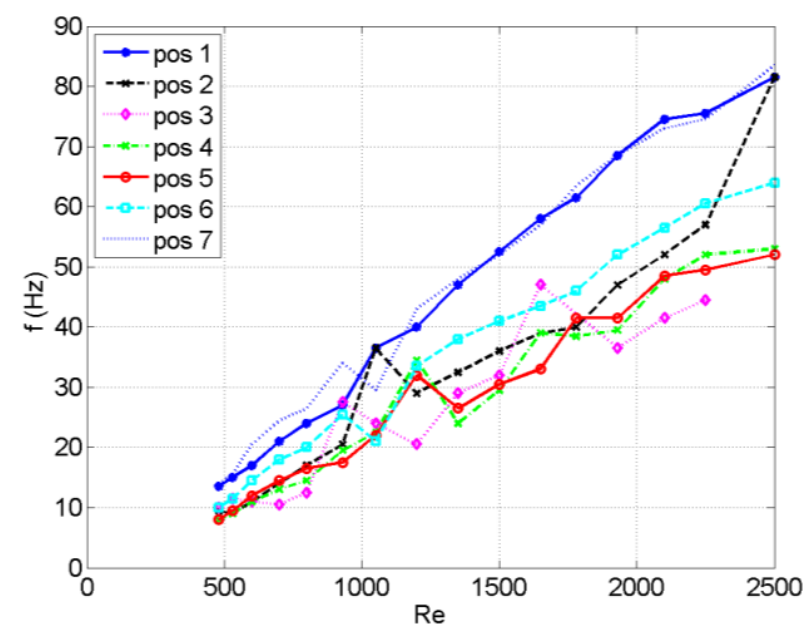

Figure 12. Relationship between the frequency and Reynolds numbers for $\mathrm{D} / \mathrm{d}=1$

\section{Conclusion}

Set of measurements of frequencies of vortex shedding were performed for combination of cylinders in staggered configuration and for 16 modes of Reynolds number. The main aim was to compare the individual positions of the secondary cylinder in the wake of the primary cylinder, and compare these positions with research [3] and [4], which were made, however, for lower Re and other D / d ratio.

The measured results show that for the D / d = 1 the position with most suppressing instabilities are 3,4 and 5, which corresponds to position $3: \mathrm{x} / \mathrm{D}=2.4$ and $\mathrm{y} / \mathrm{D}=$ 8, position 4: $\mathrm{x} / \mathrm{D}=1.95$ and $\mathrm{y} / \mathrm{D}=-0.8$ and position 5 : $\mathrm{X} / \mathrm{D}=2$ and $\mathrm{y} / \mathrm{D}=-0.6$. These points lie in the area defined in [4]. Positions 1 and 6, which cause greater disruption in the wake, respectively significantly do not affect the flow, also agrees with the research in [3] and [4]. Only the results for the 7-position will vary, which may be due to the fact that the primary and secondary cylinder behave as a single bluff body, as indicated by various authors in [5].

For $\mathrm{D} / \mathrm{d}=2.5$, it was found that the most suppressive instability positions are 2,3 and 4 where position 2 corresponding to $\mathrm{x} / \mathrm{D}=3.2$ and $\mathrm{y} / \mathrm{D}=-8$. These points also located in the area defined in [4]. For the other positions the same applies as for $\mathrm{D} / \mathrm{d}=1$. If the results are compared for $\mathrm{D} / \mathrm{d}=1$ and $\mathrm{D} / \mathrm{d}=2.5$ among them we find that the $\mathrm{D} / \mathrm{d}=1$ suppresses more formation of instability than $\mathrm{D} / \mathrm{d}=2.5$.

\section{Acknowledgements}

The work was supported by the Student Grant Competition of Czech Technical University in Prague and the Centre SGS14/058/OHK2/1T/12 for Research on multiphase flow and thermodynamic phenomena in the field of renewable sources and energy - NEW ENERGY (CZ.2.16/3.1.00/22130).

\section{References}

1. M. M. Zdravkovich, J. Fluids Eng. 99(4): 618-633 (1977)

2. H. Choi, I. Lee, W.P. Jeon., J. Kim, Annu. Rev. Fluid Mech. 40:113-39 (2008)

3. P. J. Strykowski, K. R. Sreenivasan, J. Fluid Mech. 218: 71-107 (1990)

4. Y. Hwang, H. Choi, J. Fluid Mech., 560: 465-475 (2006)

5. D. Sumner, J. Fluids and Structures, 26: 849-899 (2010)

6. Y. Yokoi, K. Hirao, EPJ Web of Conf. 45, 01098 (2013)

7. R. Vitkovicova, J. Nozicka, J. Cizek, V. Skala, EPJ Web of Conf. 92, 02110 (2015) 